\title{
Cardiovascular Burden Associated with Uremic Toxins in Patients with Chronic Kidney Disease
}

\author{
Hamid Moradi ${ }^{a}$ Domenic A. Sica ${ }^{b}$ Kamyar Kalantar-Zadeh ${ }^{a}$ \\ a University of California Irvine School of Medicine, Orange, Calif., and ${ }^{b}$ Virginia Commonwealth University \\ Health System, Richmond, Va., USA
}

\section{Key Words}

Cardiovascular $\cdot$ Chronic kidney disease $\cdot$ Indoxyl sulfate .

$p$-cresyl sulfate $\cdot$ Uremic toxins

\begin{abstract}
Background: Retention of uremic toxins in patients with chronic kidney disease (CKD) negatively affects multiple organ systems, including the cardiovascular system, resulting in significant morbidity and mortality. Alleviation of the adverse effects of uremic toxins is an important priority in the management of CKD. Scope: This review focuses on the evidence for the influence of uremic toxins on cardiovascular morbidity and mortality among patients with CKD and slowly developing uremia. The cardiovascular effects of acute kidney injury and rapidly developing azotemia are beyond the scope of this review and will not be discussed. Data on potential treatment options aimed at ameliorating the toxic effects of uremic toxins are summarized. Findings: Uremic toxins are associated with significant cardiovascular morbidity and mortality in patients with CKD. While a number of preclinical studies have detailed these effects, clinical studies directly evaluating cardiovascular outcomes consequent to the presence of uremic toxins have only recently become available. Conclusion: Uremic toxins play an important role
\end{abstract}

in the progression of cardiovascular disease in patients with CKD. Further studies are needed to better characterize the impact of these compounds on cardiovascular outcomes. Beneficial treatments are currently available that, in preliminary studies, appear to neutralize some of the adverse effects of uremic toxins. Large randomized clinical trials are needed to further determine the utility of these varied therapeutic agents.

Copyright $\odot 2013$ S. Karger AG, Basel

\section{Introduction}

Uremic illness in patients with chronic kidney disease (CKD) is primarily a consequence of inadequate removal and subsequent accumulation of organic products normally metabolized or excreted by the kidney. Accumulated uremic solutes are termed uremic toxins if they are biologically active. The accumulation of uremic toxins is associated with negative effects on almost every organ system, though most notably on the cardiovascular (CV) system $[1,2]$. In patients with $\mathrm{CKD}$, cardiovascular disease (CVD) is associated with substantial morbidity and mortality, and is a major health concern even among those in early stages of this disease. Considerable effort

\section{KARGER}

E-Mail karger@karger.com

www.karger.com/ajn
(C) 2013 S. Karger AG, Basel

0250-8095/13/0382-0136\$38.00/0
Domenic A. Sica, MD

Virginia Commonwealth University Health System Richmond, VA 23298-0160 (USA)

E-Mail dsica@msch-vcu.edu 
has gone into understanding the mechanisms responsible for such toxicity and to develop therapeutic interventions which can reduce the adverse effects of uremic toxins.

Uremic toxins are both a cause and consequence of $\mathrm{CKD}$, and their negative impact on the $\mathrm{CV}$ system is thought to be multifactorial with incompletely understood mechanisms. Identifying and understanding factors that negatively impact CV health in patients with $\mathrm{CKD}$ is crucial to the development of therapies aimed at reducing adverse $\mathrm{CV}$ outcomes. It is well known that $\mathrm{CKD}$ is a chronic pro-inflammatory state that is both a cause and a result of oxidative stress. Chronic inflammation (predominantly mediated by macrophages) and oxidative stress play a critical role in the progression of $\mathrm{CKD}$ and CKD-related CVD [3, 4]. A broad range of nontraditional risk factors also appear to play a role in the pathogenesis of CVD in patients with CKD. The net effect of these traditional and nontraditional risk factors is an increase in CV-related morbidity and mortality above and beyond that which occur in patients without CKD.

Here we review the pathophysiologic effects of uremic toxins on the incidence and progression of CVD among patients with CKD, as well as therapies aimed at prevention of CKD-related CVD.

\section{Uremic Toxins}

Retention of uremic toxins leads to an environment marked by the complex interplay between a variety of retained molecules and their negative impact on multiple organ systems. Given the complexity and multiplicity of these toxins, serum urea and creatinine are inadequate as markers for uremic toxicity since they offer only a snapshot of the entire toxin picture. Also, effective treatment of uremia will require consideration of the unique characteristics of these compounds. Furthermore, it is likely that not all uremic toxins have been identified. Hence, rational treatment strategies for uremia first require a simplified classification system for uremic toxins. The most commonly applied classification system is based on physicochemical properties of uremic toxins that affect their removal during hemodialysis [5]:

(1) Small, water-soluble molecules: The molecular weight (MW) of these compounds is generally $\leq 500 \mathrm{Da}$. These compounds may or may not display marked toxic activity.

(2) Middle molecules: These compounds have a MW of $>500 \mathrm{Da}$ and are removed only via hemodialysis employing large-pored membranes. Many compounds in this group are peptides that are toxic to multiple organ systems.

(3) Protein-bound molecules: Most compounds in this group have a low MW; however, some have characteristics similar to middle molecules. Many of these compounds are toxic and difficult to remove via hemodialysis.

In 2003, the European Uremic Toxin Work Group classified 90 compounds by this system (table 1), and more compounds have since been identified.

Alternatively, uremic toxins may be classified according to their site of origin [6] and include toxins:

(1) produced by endogenous metabolism,

(2) produced by microbial metabolism, or

(3) ingested from an exogenous source.

This classification strategy may help in identifying treatments other than extracorporeal removal. Although the majority of toxins fall into the first category, a significant number originate from microbial metabolism. Exogenous dietary toxins such as oxalate and advanced glycation end products also contribute to uremia [6]. A number of uremic toxins, including phenolic and indolic compounds, originate from protein fermentation in the large intestine following hydrolysis of polypeptide chains by proteases and peptidases. The latter leads to the production of small peptides and amino acids, which are either used by intestinal flora for growth or are further fermented into short- or branched-chain fatty acids and other potentially toxic metabolites, including ammonia, amines, thiols, phenols, and indoles. Phenols (e.g. phenylacetic acid (PAA), phenol, $p$-cresol) are generated during the partial breakdown of tyrosine and phenylalanine by intestinal facultative or obligate anaerobes. Most phenols produced in the colon are absorbed and detoxified by conjugation to sulfate compounds by the liver (e.g. $p$-cresol to $p$-cresyl sulfate; PCS) [6]. Indoles and amines are also end products of metabolism in the colon. Breakdown of tryptophan results in production of indolic compounds, and decarboxylation of lysine and ornithine leads to production of simple amines. Indoles are absorbed and metabolized to the sulfate form by the liver (e.g. indoxyl sulfate; IS). Amines can be detoxified by monoamine and diamine oxidases in the liver or colonic mucosa or can be renally excreted [6].

CKD is classified into 5 stages based on glomerular filtration rate (GFR) [7]. Given the difficulties in accurately measuring GFR in routine clinical practice, GFR is estimated (eGFR) on the basis of serum creatinine levels combined with demographic and anthropometric parameters [8]. Estimated GFR based on serum creatinine has been shown to be poorly correlated with concentra- 
Table 1. Uremic toxins classified by physicochemical properties

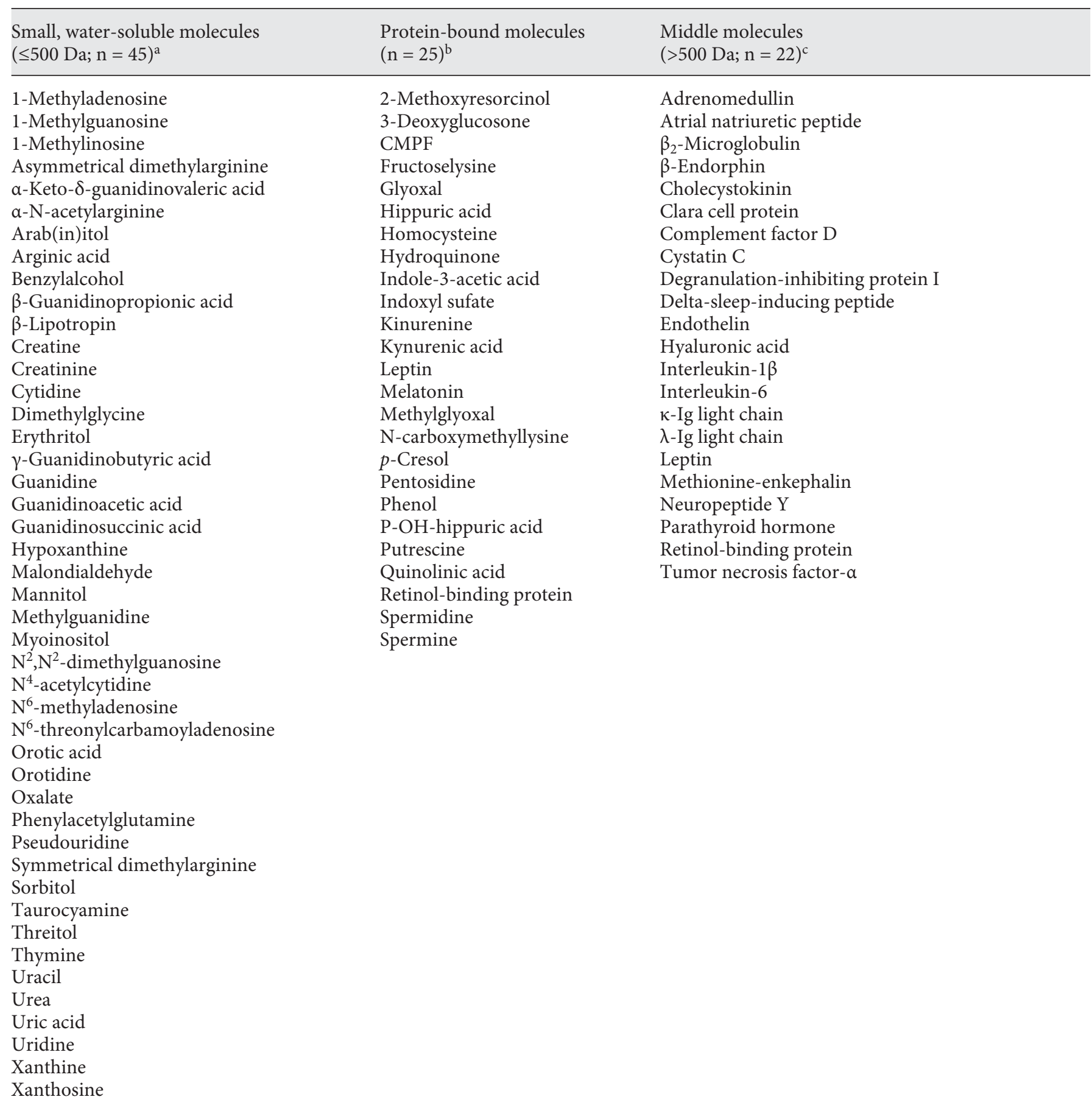

Reprinted with permission from Macmillan Publishers: Copyright ${ }^{\complement}$ Kidney International, Vanholder et al. [5].

a These compounds are effectively removed by any dialysis strategy.

b These compounds are, with the sole exception of hippuric acid, difficult to remove regardless of the type of membrane used for hemodialysis [62]. They are more efficiently removed by peritoneal dialysis, which uses membranes with a large pore diameter [63].

${ }^{c}$ These compounds are effectively removed only by membranes with a large pore size, such as those used in peritoneal dialysis. 
tions of uremic toxins in patients with different stages of $\mathrm{CKD}$, calling into question its utility in evaluating the accumulation of different solutes during the progression of CKD [9].

\section{Association of CKD and CVD}

Patients with CKD are at high risk for CVD even when kidney function is only minimally impaired and are more likely to succumb to CVD than to progress to kidney failure regardless of their CKD stage [10-15]. Overall, approximately half of all deaths among patients with $\mathrm{CKD}$ are a direct result of CVD. Importantly, the risk and severity of CVD increases with each incremental increase in severity of CKD, particularly among younger patients. Patients with CKD have a 10- to 20-fold increase in risk of cardiac death versus patients without CKD [16].

The association of CKD with CVD may be explained by the higher prevalence of nontraditional and traditional risk factors for CVD among patients with CKD compared with the general population $[11,17]$. Anemia, proteinuria, increases in levels of pro-inflammatory cytokines, oxidative stress, hyperhomocysteinemia, abnormal calcium and phosphate metabolism, and accumulation of uremic toxins constitute nontraditional risk factors, while traditional risk factors include hypertension, diabetes, advanced age, dyslipidemia, and tobacco use. Additional risk factors seen in hemodialysis patients include bacteremia, extracellular fluid overload, and glycemic load. The relative contributions of individual risk factors are not well understood and may be dependent on CKD stage [17].

Accumulation of uremic toxins consequent to the progressive deterioration of glomerular filtration in patients with CKD has a deleterious effect on multiple body functions (e.g. cardiac failure, hypertension, fluid overload) [2]. Multiple studies have implicated elevated concentrations of a variety of uremic toxins, including indoxyl sulfate, $p$-cresol, $p$-cresyl sulfate, PAA, blood urea nitrogen, and uric acid in the CVD seen in CKD patients [18-31]. The association of elevated uric acid concentrations with CVD in these epidemiologic studies has been corroborated by results of prospective clinical trials involving allopurinol, which decreases serum uric acid levels by inhibiting xanthine oxidase $[32,33]$. These trials, which included patients with asymptomatic hyperuricemia and patients with reduced renal function as measured by eGFR, showed that allopurinol slows progression of renal disease and reduces risk of CV events $[32,33]$.
Studies in animal models have suggested that elevated concentrations of marinobufagenin (MBG), which belongs to a class of circulating steroid compounds called the bufadienolides, may potentially play a role in the CVD associated with CKD [34]. Administration of MBG has been shown to result in changes in cardiac parameters that resemble those seen following partial nephrectomy [35]. These changes could be reversed by immunization against MBG, supporting the notion that controlling elevated levels of MBG in patients with CKD may improve cardiac symptoms [35].

In addition, an association between arterial calcification and increased CVD-related mortality has emerged among patients with CKD. The presence and extent of vascular calcifications are strong predictors of CVD-related and all-cause mortality in end-stage renal disease (ESRD) patients on maintenance hemodialysis. It is unclear whether enhanced arterial stiffness is a risk factor contributing to the development of CVD or a marker of more established disease; however, studies suggest that measurement and serial monitoring of arterial parameters related to vascular structure and function may offer some promise in risk assessment and development of therapeutic strategies [36].

In summary, substantial evidence supports the relationship between CKD and poor CVD-related outcomes. Both traditional and nontraditional risk factors are responsible for these associations. Mechanisms for measuring the relative contributions of nontraditional risk factors are being explored. Accumulating data suggest that uremic toxins play an important role in the morbidity and mortality from CVD among patients with CKD. Identification of these toxins and defining the pathobiologic mechanisms by which they cause CVD are prerequisites for development of therapeutic interventions to alleviate poor CV outcomes in patients with CKD.

In the following sections, we review the evidence indicating that uremic toxins contribute substantially to the development and severity of CVD among patients with CKD. In addition, we discuss mechanisms by which these compounds impact CVD, as well as potential therapeutic strategies for mitigating their toxic effects.

\section{Cardiovascular Effects of Uremic Toxins}

Although uremic toxins affect almost every organ system, this review will focus on the impact of uremic toxins on markers for CVD and on CVD-related outcomes in patients with CKD. Treatment of traditional CVD risk 
Table 2. Role of uremic toxins in cardiovascular damage

\begin{tabular}{|c|c|c|c|}
\hline \multicolumn{4}{|c|}{ Uremic toxins result in vascular damage via } \\
\hline Leukocyte activation & Glycocalyx and endothelium & Smooth muscle cells & Platelets \\
\hline $\begin{array}{l}\text { - Stimulates TNF- } \alpha \\
\text { production } \\
\text { - Increases oxidative stress } \\
\text { - Activates NF- } \kappa \mathrm{B} \\
\text { - Upregulates cell adhesion } \\
\text { molecules } \\
\text { - Increases IL-6 leading to } \\
\text { aortic calcification }\end{array}$ & $\begin{array}{l}\text { - Disruption of the endothelial } \\
\text { glycocalyx contributes to } \\
\text { increased microvascular } \\
\text { permeability resulting in } \\
\text { systemic vascular dysfunction } \\
\text { - Increases PAI-1 and von } \\
\text { Willebrand factor } \\
\text { - Decreases tissue plasminogen } \\
\text { factor } \\
\text { - Dysregulates vascular tone } \\
\text { - Produces ROS } \\
\text { - Inhibits NO production } \\
\text { - Promotes cellular senescence }\end{array}$ & $\begin{array}{l}\text { - Increases proliferation } \\
\text { of vascular smooth } \\
\text { muscle cells leading } \\
\text { to aortic calcification } \\
\text { and wall thickening }\end{array}$ & $\begin{array}{l}\text { - Activated platelets seen in uremic } \\
\text { patients support thrombosis, } \\
\text { which may result from exposure } \\
\text { of PS on the outer membrane of } \\
\text { the platelet in a process mediated } \\
\text { by caspase-3 activity } \\
\text { - Enhanced caspase- } 3 \text { activity has } \\
\text { been documented in uremic } \\
\text { patients } \\
\text { - Inhibition of caspase- } 3 \text { activity } \\
\text { results in decreased PS exposure } \\
\text { on the outer membrane of } \\
\text { platelets }\end{array}$ \\
\hline
\end{tabular}

factors in this population only partially reduces CVD-related morbidity and mortality compared with the general population. Thus, it is important to identify and describe additional mechanisms that contribute to atherogenesis and CVD in patients with CKD. Uremic toxins represent one area of nontraditional CVD risk factors unique to patients with CKD. The effects of uremic toxins can be described in terms of their impact on cell systems associated with CVD. The main cell types involved in the CVD associated with CKD include leukocytes, endothelial cells, vascular smooth muscle cells (VSMC), and platelets (table 2) [2,37]. The involvement of the different uremic toxins in the four major domains of CVD (atherosclerosis, myocardial disease, valvular disease, arrhythmias) is summarized in table 3.

\section{Uremic Toxins and Leukocytes}

Uremic toxins inhibit leukocyte function in CKD patients by reducing their basal activation and blunting their response upon stimulation. The former effect may result in infection; the latter has been linked to malnutrition, chronic microinflammation, and atherosclerosis. Guanidine compounds have been implicated in CV damage given their stimulatory effect on leukocytes, leading to enhancement of tumor necrosis factor (TNF)- $\alpha$ production and increased leukocyte-induced oxidative stress [1]. In addition, PCS has been shown to enhance susceptibility to vascular damage by promoting enhanced oxidative activity in leukocytes [22]. The uremic toxin ho- mocysteine activates nuclear factor-kappa B (NF- $\mathrm{BB})$ in macrophages, resulting in increased superoxide anion levels and oxidative stress, which can be reversed by administration of folic acid.

\section{Uremic Toxins and Leukocyte-Endothelial Cell Interaction}

Leukocyte-endothelial interactions are an important step in the development of atherosclerosis. Cell adhesion molecules (e.g. intercellular cell adhesion molecule-1 (ICAM-1) and vascular cell adhesion molecule-1), together with E-selectin, are upregulated to mediate monocyte and macrophage infiltration into atherosclerotic lesions. IS was recently shown to enhance leukocyte-endothelial interactions through upregulation of E-selectin. This likely occurs via the JNK-NF- $\mathrm{kB}$-dependent pathway and represents a novel mechanism for the induction of vascular pro-inflammatory processes observed in CKD [38].

\section{Uremic Toxins and Chronic Inflammation}

It has been amply demonstrated that $\mathrm{CKD}$ is associated with a state of chronic inflammation, as evidenced by elevated concentrations of various pro-inflammatory cytokines such as IL-6 and altered concentrations of acutephase proteins such as C-reactive protein (CRP). Given the critical role chronic inflammation plays in the development and progression of CVD, uremia-associated pro- 
Table 3. Involvement of uremic toxins in the major domains of cardiovascular disease

\begin{tabular}{|c|c|c|c|c|}
\hline \multirow[t]{2}{*}{ Uremic toxin } & \multicolumn{4}{|c|}{ Cardiovascular disease domain } \\
\hline & $\begin{array}{l}\text { athero- } \\
\text { sclerosis }\end{array}$ & $\begin{array}{l}\text { myocardial } \\
\text { disease }\end{array}$ & $\begin{array}{l}\text { valvular } \\
\text { disease }\end{array}$ & arrhythmias \\
\hline Advanced glycation end products & $\mathrm{X}$ & & & \\
\hline Advanced oxidation protein products & $\mathrm{X}$ & & & \\
\hline Angiogenin-DIP I & $\mathrm{X}$ & & & \\
\hline$\beta_{2}$-Microglobulin & $\mathrm{X}$ & $\mathrm{X}$ & & \\
\hline \multicolumn{5}{|l|}{ Complement factor D } \\
\hline Cytokines & $\mathrm{X}$ & $\mathrm{X}$ & $?$ & $?$ \\
\hline \multicolumn{5}{|l|}{ Dinucleotide polyphosphates $\left(\mathrm{Np}_{\mathrm{x}} \mathrm{N}\right)$} \\
\hline $\begin{array}{l}\text { Guanidino compounds (e.g. methylguanidine, } \\
\text { asymmetrical dimethylarginine) }\end{array}$ & $\mathrm{X}$ & & & \\
\hline Homocysteine & $\mathrm{X}$ & & & \\
\hline Indoxyl sulfate & $\mathrm{X}$ & & & \\
\hline Leptin & $\mathrm{X}$ & & & \\
\hline Oxalic acid/oxalate & $\mathrm{X}$ & & & \\
\hline$p$-Cresol/p-cresyl sulfate & $\mathrm{X}$ & & & \\
\hline Parathyroid hormone & & & $\mathrm{X}$ & \\
\hline PAA & $\mathrm{X}$ & & & \\
\hline Phosphate & & & $\mathrm{X}$ & \\
\hline
\end{tabular}

inflammatory cytokines are considered a major component of uremic toxicity. In a study of 125 patients with CKD stage 2-5, IL-6 was independently associated with CKD stage and plasma levels of CRP and albumin [4]. Follow-up studies of these patients showed that plasma IL-6 levels also significantly predicted CVD and all-cause mortality after adjusting for potential confounders. Furthermore, IL- 6 was superior to CRP, albumin, or TNF- $\alpha$ in predicting mortality in this patient cohort [4]. Another group of uremic toxins, the guanadino compounds (particularly symmetric dimethylarginine) induce production of reactive oxygen species (ROS) and pro-inflammatory markers (mainly related to microinflammation and leukocyte activation) leading to chronic inflammation [39]. In addition, the surface expression of CD-14, a marker for monocyte differentiation, was increased by all guanadinos except guanidine. Therefore, these compounds contribute to monocyte $\mathrm{CD}$ expression that may result in enhanced differentiation and endothelium adhesion capacity of these cells and hence ongoing chronic inflammation [39]. Symmetric dimethylarginine is also associated with activation of NF- $\kappa B$ in addition to increased IL- 6 and TNF- $\alpha$ expression. Furthermore, AGE accumulation in the plasma of uremic patients has been associated with oxidative stress, which can lead to chronic inflammation and CVD.

\section{Uremic Toxins and Endothelial Cell Function}

Endothelial cell integrity and function are critical to the prevention of atherosclerosis, and therefore endothelial cell injury and dysfunction are major steps in the development and progression of CVD. There are several mechanisms through which endothelial cells protect against CVD, including endothelial cell function, endothelial cell integrity and barrier properties and endothelial cell repair. In CKD, endothelial dysfunction can be a result of oxidative stress, chronic inflammation, and production of ROS [1]. Furthermore, several uremic compounds have been shown to inhibit endothelial proliferation, potentially contributing to endothelial cell dysfunction [39]. Moreover, impairment of endothelial cell function among patients with CKD is associated with a pro-coagulant environment at the surface of these cells (as illustrated by increases in plasminogen activator inhibitor-1 and von Willebrand factor and decreases in tissue plasminogen activator), as well as dysregulation of vascular tone due to inhibition of endothelial iNOS. While endothelial function is dependent on NO, the production of $\mathrm{NO}$ is decreased in patients with CKD. Studies in mice have shown that CKD is associated with an increased concentration of the uremic toxin asymmetric di- 
methylarginin, which alters endothelial cell function in cerebral circulation, perhaps via a decrease in NO production. This study did not identify any changes in arteriolar structure, mechanics, or composition [40].

Another important mechanism for CKD-associated NO deficiency is an increase in oxidative stress. Uremic toxins increase the generation of ROS that cause oxidative stress. This is the case for IS, which has a major deleterious effect on the endothelial system. This compound has been shown to inhibit NO production and reduce cell viability in vitro via production of ROS in vascular endothelial cells. The deleterious role of oxidative stress in this process was confirmed via use of antioxidants that prevented ISinduced ROS production and preserved cell viability [41]. A subsequent study showed that IS also upregulated the expression of ICAM-1 and MCP-1 via activation of the NF- $\kappa B$ pathway, which further reinforced the association between IS and development of CVD [42, 43]. Furthermore, IS has been shown to negatively influence protective properties of endothelial cells, such as migration and tube formation, by depleting NO bioavailability [43, 44]. This mechanism was further confirmed through the use of an NO donor, S-nitroso-N-acetyl-DL-penicillamine, which reversed these effects [44]. Interestingly, the potential deleterious impact of IS on the CV system appears to be concentration dependent. At concentrations found in CKD patients, the pro-oxidant effects of IS seem to predominate, leading to increased CVD. However, at normal serum concentrations, IS appears to eliminate hydroxyl radicals and displays radical scavenging activity, properties which may have a protective effect [45].

In addition, IS has been associated with increased oxidation of albumin, which is a marker for oxidative stress in systemic circulation. In a rat model of CKD, the ratio of oxidized albumin positively correlated with serum IS levels, suggesting an increase in oxidative stress in the systemic circulation [46]. The ratio was reduced in the group that received AST-120 (an orally administered intestinal adsorbent) [46]. In addition, IS increased the generation of ROS in a dose- and time-dependent manner [46].

Another uremic toxin that has been shown to cause vascular damage through inhibition of iNOS leading to reduction of endogenous $\mathrm{NO}$ and vascular dysfunction is PAA. Moreover, this compound inhibits the activity of $\mathrm{Ca}^{2+}$-ATPase, resulting in elevated intracellular $\mathrm{Ca}^{2+}$ concentration that can lead to cell death and further endothelial dysfunction.

Barrier function is an important property of vascular endothelium. Barrier dysfunction is a result of gaps between adjacent cells from loss of intercellular junctions.
A recent bovine study reported disruption of intercellular contacts on adherens junctions of epithelial cells following IS treatment. Pretreatment with U0126, a highly specific inhibitor of MEK1/2, prevented this disruption [47].

Another mechanism of disruption in endothelial cell integrity and dysfunction that is associated with the development of CVD is cellular senescence. This process involves the arrest of cells in a mitotic diploid form. Endothelial cell senescence is induced by many factors, including oxidative stress and the accumulation of uremic toxins. Administration of IS and asymmetric dimethylarginine to hypertensive rats has been shown to cause increased expression of aortic cell senescence molecules and aortic calcification $[48,49]$.

Endothelial repair mechanisms that can be mediated via proliferation or homing of circulating progenitor cells play a critical role in the health of endothelial cells and prevention of CVD. It is of note that the effectiveness of endothelial repair mechanisms is reduced in patients with $\mathrm{CKD}$, possibly leading to reduced vascular integrity and increased risk of CVD. These circulating microparticles represent a new biomarker for CVD in uremia [50]. Both IS and PCS, at concentrations typically found in uremia, have been shown to reduce endothelial proliferation and repair in vitro and thus negatively affect endothelial repair mechanisms (this effect was dose dependent for PCS). The presence of albumin did not alter the inhibitory effect of these compounds on endothelial proliferation; however, the deficiency in endothelial repair was less severe in the presence of albumin.

Oxalate, another uremic toxin, has also been associated with negative CV-related consequences most likely via negative effects on endothelial cell repair and function. Oxalate increases intracellular calcium concentrations in endothelial cells and prevents re-endothelialization [51]. It also inhibits endothelial cell replication and migration, which play a role in inhibition of atherosclerosis. The inhibitory effect of oxalate was found to be both dose and time dependent [52].

\section{Uremic Toxins and Vascular Smooth Muscle Cells}

Uremic toxins have been shown to promote proliferation of VSMC and their subsequent transformation into osteoblast-like cells capable of producing a matrix of bone collagen and noncollagenous proteins. This matrix can subsequently mineralize as a consequence of dysregulated mineral metabolism, leading to aortic wall thickening and calcification $[13,53]$. 
Yamamoto et al. [54] were the first to demonstrate that IS causes VSMC proliferation via activation of the p44/42 mitogen-activated protein kinase pathway in vitro. This pathway has been shown previously to be activated by growth factors, angiotensin II, erythropoietin, and other uremic toxins such as homocysteine and uric acid. The deleterious effect of IS on VSMC was confirmed using probenecid, an inhibitor of IS secretion. Treatment with probenecid resulted in IS retention, which inhibited VSMC proliferation [54]. Similar results were also seen in a subsequent study [55]. IS has also been shown to promote aortic calcification and wall thickening in hypertensive rats [56].

While VSMC dysfunction plays a key role in CVD, cardiac and aortic abnormalities were independently associated with the extent of endothelial and VSMC dysfunction and the severity of kidney disease in a mouse model of CKD [57]. In a longitudinal study evaluating changes in vascular stiffness throughout the progression of $\mathrm{CKD}$, mice with CKD quickly developed a significant increase in aortic stiffness, supporting the hypothesis that CKD accelerates normal age-related changes in aortic function [57]. These results suggested that calcification may not be the only mechanism responsible for vascular stiffness in early-stage CKD [57].

\section{Uremic Toxins and Platelets}

Activation of platelets in CKD patients undergoing hemodialysis has been extensively studied. Activated platelets may contribute to the CVD burden in patients with ESRD given their involvement in thrombus formation after being retained on the dialysis membranes $[37,58]$. Platelets from uremic patients show significantly higher coagulation activity than do platelets from normal individuals. This activity is partly due to the enhanced exposure of phosphatidylserine to the outer leaflet of platelet membranes in a process mediated by caspase- 3 [37]. Caspase- 3 activity is significantly higher in the platelets of uremic patients and its selective inhibition reduces phosphatidylserine exposure, highlighting the central role for caspase- 3 activation in platelet activation and therefore the pathogenesis of CVD in patients with uremia [37].

\section{Uremic Toxin-Associated CVD: Clinical Evidence}

More recently, complementary clinical studies have been conducted examining the role of uremic toxins on $\mathrm{CV}$ outcomes in patients with CKD. These studies rein- force the findings of the preclinical investigations discussed previously herein. In a cohort of 100 maintenance hemodialysis patients, Meijers et al. [59] studied the relationship between PCS and blood markers of endothelial cell dysfunction (soluble P-selectin and endothelial microparticles), as well as the direct effects of $p$-cresol and PCS on endothelial cells. While free serum $p$-cresol concentrations were positively associated with circulating endothelial microparticles, no association was found with soluble P-selectin. These findings were further confirmed in vitro (via human umbilical vein endothelial cells) as PCS was shown to induce a dose-dependent increase in endothelial microparticles. This effect was reduced in the presence of albumin. The authors concluded that PCS alters endothelial function in patients on hemodialysis [59].

Clinical studies in patients with CKD confirm the preclinical findings regarding the effects of IS on the CV system. IS concentrations have been shown to be independently and positively associated with pentosidine (an AGE compound), creatinine, and protein catabolic rate, which are risk factors for atherosclerosis in patients on maintenance hemodialysis. IS was also found to be independently and negatively associated with high-density lipoprotein cholesterol [19]. Barreto et al. [18] investigated the association between vascular calcification, vascular stiffness, mortality, and serum IS concentrations in a cohort of patients with CKD and found an inverse relationship between baseline IS levels and renal function and a direct relationship with aortic calcification. Survival analyses found that the highest IS tertile was a strong predictor for death even after adjustments for age, gender, diabetes, albumin, hemoglobin, phosphate, and aortic calcification [18]. Chiu et al. [21] studied patients with type 2 diabetes and evaluated the association between IS and PCS concentrations and coronary artery disease (CAD) in a hospital-based case-control format. Serum IS and PCS concentrations were significantly higher in patients with both type 2 diabetes and substantial CAD as compared to diabetic and nondiabetic patients without CAD [21]. Both compounds were independently and positively associated with the presence of CAD. Increased concentrations of these compounds were associated with deterioration of renal function and coronary atherosclerosis [21]. Patients with significant coronary artery stenosis have also been shown to have higher serum PCS levels, which were independently associated with the presence and severity of CAD [23]. In the first clinical study to evaluate $p$-cresol levels and mortality, Bammens et al. [60] found an independent relationship between free se- 
rum $p$-cresol concentrations and all-cause mortality in hemodialysis patients followed for 34 months. A subsequent analysis found that free serum $p$-cresol concentrations were also significantly associated with time to first $\mathrm{CV}$ event. In multivariate analysis, free $p$-cresol concentrations were found to be significantly associated with CVD among patients who did not have diabetes [24]. Liabeuf et al. [22] also found an independent association between free PCS concentrations and mortality in patients at different stages of CKD. Baseline total and free PCS concentrations were inversely related to renal function and positively associated with vascular calcification. Although both free and total $p$-cresol concentrations were higher in more severe $\mathrm{CKD}$, only free concentrations were associated with risk of death [22].

PAA has been shown to inhibit iNOS expression and plasma membrane calcium ATPase. A study evaluating the effect of PAA on the vascular system in patients with stage $5 \mathrm{CKD}$ on maintenance hemodialysis found a significant correlation between the PAA concentration and vascular dysfunction as measured by the reflective index obtained from digital photoplethysmography [26].

In summary, strong evidence substantiating the toxic effects of uremic toxins in the development and progression of CVD have been corroborated by clinical studies. Strategies for prevention of CVD-related morbidity and mortality should focus on mechanisms for removing uremic toxins before negative outcomes occur, as well as therapeutic options for ameliorating their effects. One area of particular interest could be the development of clinical trials that confirm the effectiveness (as measured by superior CV outcomes) of large-pore dialyzer membranes for facilitated removal of uremic toxins. In the following section, we review information on therapeutic options for mitigation of the adverse effects of uremic toxins.

\section{Therapeutic Interventions}

Inhibiting uremic toxins has been shown to reduce toxin-associated pro-inflammatory processes, endothelial dysfunction, and aortic calcification. Methods to treat uremia include enhancement of the removal of uremic toxins through hemodialysis or peritoneal dialysis and use of pharmacologic approaches to interfere with their effects [1]. The ultimate aim of renal replacement therapy is the removal of uremic toxins and the preservation of residual renal function, which has been associated with enhanced survival [61].
As discussed earlier, uremic toxins are classified on the basis of their physicochemical properties [2]. Of these, protein-bound molecules are the most difficult to remove using currently available dialysis strategies $[2,62]$. Comparison of the effectiveness of 3 different types of highflux and low-flux membranes in removing a variety of uremic toxins through hemodialysis revealed that removal of protein-bound or lipophilic solutes was, almost without exception, highly inefficient, regardless of the type of membrane used [62]. Peritoneal membranes, which have fewer pores of greater diameter, more efficiently remove middle molecules and protein-bound molecules [63]. This translates into reduced mortality during the first few years of peritoneal dialysis as well as better preservation of residual renal function compared with hemodialysis $[63,64]$. Multiple studies have documented lower plasma levels of $\beta_{2}$-microglobulin, advanced oxidation protein products, and total as well as free $p$-cresol in patients on peritoneal dialysis compared with those on hemodialysis, with the differences remaining significant for $\beta_{2}$-microglobulin and $p$-cresol even after accounting for differences in residual renal function between the 2 groups of patients [64]. However, both peritoneal dialysis and hemodialysis are only partially effective in removing many of the uremic toxins [2, 63, 64]. This underscores the urgent need for development of alternative techniques for effective removal of all uremic toxins. Encouraging results have been reported by a recent study, which has revealed that online hemodiafiltration using higher convective volumes reduces all-cause mortality by $30 \%$ compared with traditional dialysis [65]. This survival advantage may be due to a more effective removal of uremic toxins which are poorly dialyzable using conventional hemodialysis. Meanwhile, pharmacologic interventions have also been shown to reduce or prevent toxicity from these uremic compounds.

The therapeutic advantages of several inert binding compounds have been reported (table 4). These agents include sevelamer (a nonabsorbed poly-allylamine hydrochloride polymer) and AST-120 (an intestinal adsorbent) (fig. 1).

Sevelamer has been shown to decrease levels of CRP and to alleviate systemic inflammation by reducing endotoxemia in an experimental model of CKD [66]. Sevelamer was associated with a 78\% reduction in CRP levels and a parallel decrease in endotoxemia after 6 months in 20 patients on hemodialysis [66].

AST-120 has been observed to reduce levels of creatinine and urea, as well as all-cause mortality, in patients with CKD. Currently, it is used to delay the initiation of 
Table 4. Potential treatments for the management of CKD

\begin{tabular}{lll}
\hline Treatment & Description & Clinical effects \\
\hline Sevelamer & $\begin{array}{l}\text { Nonabsorbed poly(allylamine } \\
\text { hydrochloride) polymer }\end{array}$ & $\begin{array}{l}78 \% \text { reduction in CRP following } 6 \text { months of treatment in hemodialyzed patients } \\
{[66]}\end{array}$ \\
\hline AST-120 & Intestinal adsorbent & $\begin{array}{l}\text { Increased vasodilation, decreased indoxyl sulfate levels, and decreased markers of } \\
\text { oxidative stress following 24 weeks of treatment in patients with CKD [20] }\end{array}$ \\
\hline
\end{tabular}

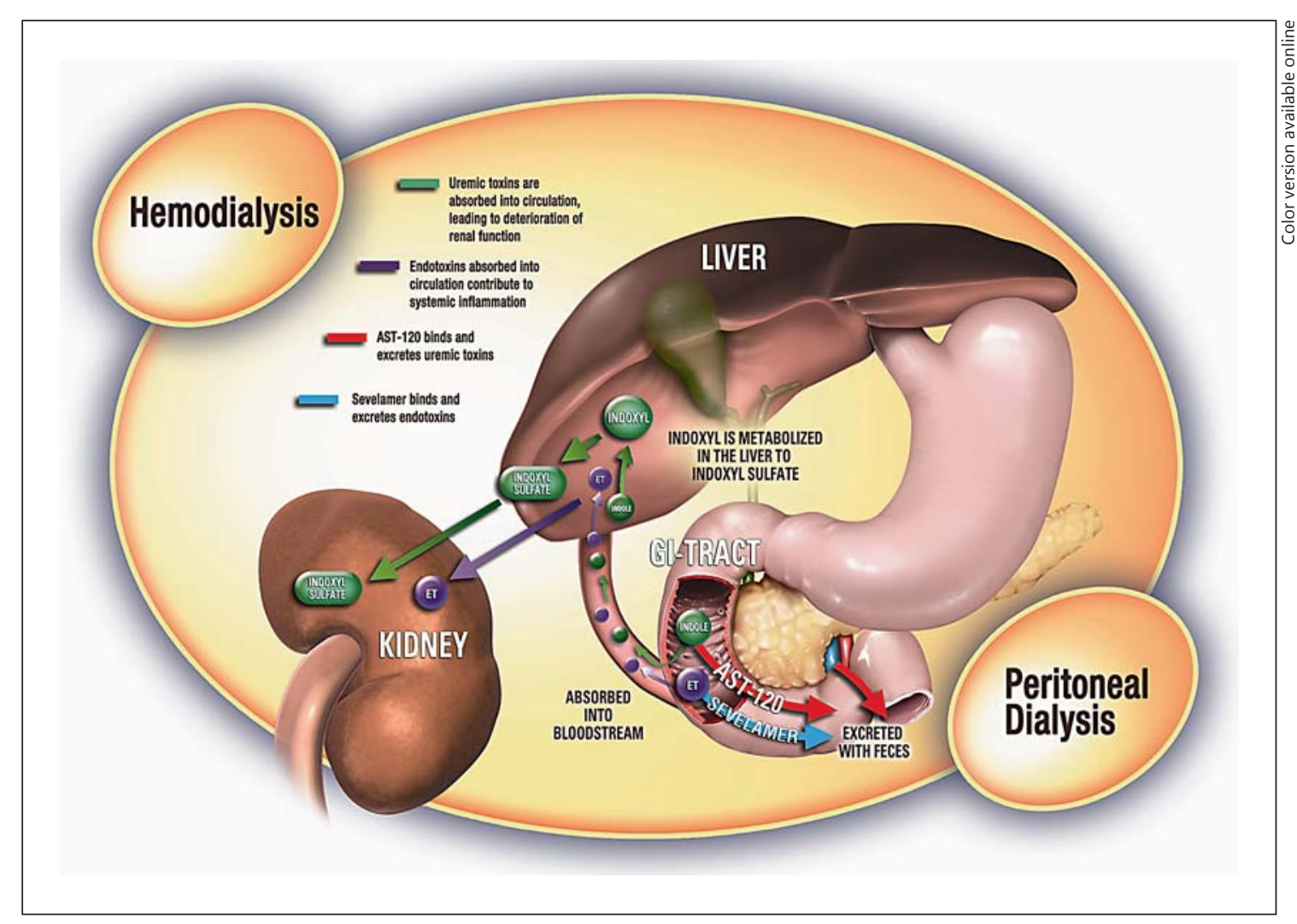

Fig. 1. Options for removal of toxins from the body $[20,66,70]$. ET = Endotoxin; GI = gastrointestinal.

hemodialysis [67]. AST-120 significantly reduced atherosclerosis and altered lesion characteristics in mice with renal damage, independent of total cholesterol, blood pressure, or creatinine clearance. It also decreased expression of monocyte chemoattractant protein-1, TNF- $\alpha$, and IL$1 \beta$ messenger RNA [67]. In another study, AST-120 treatment for 24 weeks was associated with a significant increase in flow-mediated endothelium-level vasodilation in the brachial artery of 40 patients with CKD. There was also a decrease in plasma concentration of IS. Independent predictors for improved endothelium-level vasodila- tion included presence of diabetes and CRP. Overall, AST120 improved endothelial dysfunction in patients with CKD and was associated with a decrease in markers of oxidative stress [20]. Research suggests that AST-120 might reduce arterial stiffness and intima-media thickness (which are related to CAD) in nondiabetic patients before hemodialysis. Treatment with AST-120 for 24 months decreased carotid artery intima-media thickness and pulsewave velocity (a measure of arterial stiffness) in patients with nondiabetic CKD when compared with patients who did not receive AST-120 [68]. Symptoms and outcomes of 
heart failure were improved in patients with congestive heart failure and moderate CKD after treatment with AST-120 in addition to conventional therapy for 24 months. In addition, of those who required hospital admission, the length of hospital stay and number of hospital admissions decreased significantly after treatment with AST-120 when compared with the 2-year period prior to initiation of therapy. Improvements in renal function indices, atrial natriuretic peptide, edema, and cardiothoracic ratio have also been reported [69].

\section{Conclusions}

Uremic toxins have a clear role in the progression of renal dysfunction and CVD in patients with CKD. The vast majority of uremic toxins are either highly protein bound or middle MW compounds, making their removal via standard hemodialysis and peritoneal dialysis dif- ficult. Additional studies are needed to further delineate the impact of uremic toxins on outcomes such as CVrelated adverse events and mortality in patients with CKD. The availability of promising agents, currently in phase III trials, aimed at reducing uremic toxins will offer further therapeutic options for patients with CKD.

\section{Acknowledgments}

H.M. and K.K.-Z. are supported by National Institutes of Health grants F32-DK082130 and K24-DK091419, respectively. Editorial assistance, including for figure 1, was provided by ApotheCom (Yardley, Pa., USA).

\section{Disclosure Statement}

Editorial assistance by ApotheCom was funded by Mitsubishi Tanabe Pharma Corporation.

\section{References}

1 Vanholder R, Baurmeister U, Brunet P, Cohen G, Glorieux G, Jankowski J: A bench to bedside view of uremic toxins. J Am Soc Nephrol 2008;19:863-870.

2 Vanholder R, Van Laecke S, Glorieux G: What is new in uremic toxicity? Pediatr Nephrol 2008;23:1211-1221.

3 Manabe I: Chronic inflammation links cardiovascular, metabolic and renal diseases. Circ J 2011;75:2739-2748.

4 Barreto DV, Barreto FC, Liabeuf S, et al: Plasma interleukin-6 is independently associated with mortality in both hemodialysis and predialysis patients with chronic kidney disease. Kidney Int 2010;77:550-556.

5 Vanholder R, De Smet R, Glorieux G, Argiles A, Baurmeister U, Brunet P, Clark W, Cohen G, De Deyn PP, Deppisch R, Descamps-Latscha B, Henle T, Jorres A, Lemke HD, Massy ZA, Passlick-Deetjen J, Rodriguez M, Stegmayr B, Stenvinkel P, Tetta C, Wanner C, Zidek W: Review on uremic toxins: classification, concentration, and interindividual variability. Kidney Int 2003;63:1934-1943.

-6 Evenepoel P, Meijers BK, Bammens BR, Verbeke K: Uremic toxins originating from colonic microbial metabolism. Kidney Int Suppl 2009;S12-S19.

7 National Kidney Foundation: Kidney disease outcome quality initiative (K/DOQI) advisory board: K/DOQI clinical practice guidelines for chronic kidney disease: evaluation, classification, and stratification: kidney disease outcome quality initiative. Am J Kidney Dis 2002;39:S17-S31.
8 Levey AS, Stevens LA, Schmid CH, et al: A new equation to estimate glomerular filtration rate. Ann Intern Med 2009;150:604612.

$\checkmark$ Eloot S, Schepers E, Barreto DV, et al: Estimated glomerular filtration rate is a poor predictor of concentration for a broad range of uremic toxins. Clin J Am Soc Nephrol 2011;6: 1266-1273.

10 Weiner DE, Tighiouart H, Amin MG, Stark PC, MacLeod B, Griffith JL, Salem DN, Levey AS, Sarnak MJ: Chronic kidney disease as a risk factor for cardiovascular disease and allcause mortality: a pooled analysis of community-based studies. J Am Soc Nephrol 2004; 15:1307-1315.

11 Sarnak MJ, Levey AS, Schoolwerth AC, Coresh J, Culleton B, Hamm LL, McCullough PA, Kasiske BL, Kelepouris E, Klag MJ, Parfrey P, Pfeffer M, Raij L, Spinosa DJ, Wilson PW, American Heart Association Councils on Kidney in Cardiovascular Disease, High Blood Pressure Research, Clinical Cardiology, and Epidemiology and Prevention: Kidney disease as a risk factor for development of cardiovascular disease: a statement from the American Heart Association Councils on Kidney in Cardiovascular Disease, High Blood Pressure Research, Clinical Cardiology, and Epidemiology and Prevention. Hypertension 2003;42:1050-1065.

$\checkmark 12$ Szczech LA, Best PJ, Crowley E, Brooks MM, Berger PB, Bittner V, Gersh BJ, Jones R, Califf $\mathrm{RM}$, Ting $\mathrm{HH}$, Whitlow PJ, Detre KM, Holmes D, Bypass Angioplasty Revascular- ization Investigation (BARI) Investigators: Outcomes of patients with chronic renal insufficiency in the bypass angioplasty revascularization investigation. Circulation 2002; 105:2253-2258.

13 Schiffrin EL, Lipman ML, Mann JF: Chronic kidney disease: effects on the cardiovascular system. Circulation 2007;116:85-97.

$\checkmark 14$ Keith DS, Nichols GA, Gullion CM, Brown JB, Smith DH: Longitudinal follow-up and outcomes among a population with chronic kidney disease in a large managed care organization. Arch Intern Med 2004;164:659-663.

15 Go AS, Chertow GM, Fan D, McCulloch CE, Hsu CY: Chronic kidney disease and the risks of death, cardiovascular events, and hospitalization. N Engl J Med 2004;351:1296-1305.

16 Jindal K, Chan CT, Deziel C, Hirsch D, Soroka SD, Tonelli M, Culleton BF: Hemodialysis clinical practice guidelines for the Canadian Society of Nephrology. J Am Soc Nephrol 2006;17:S1-S27.

17 Rucker D, Tonelli M: Cardiovascular risk and management in chronic kidney disease. Nat Rev Nephrol 2009;5:287-296.

18 Barreto FC, Barreto DV, Liabeuf S, Meert N, Glorieux G, Temmar M, Choukroun G, Vanholder R, Massy ZA, European Uremic Toxin Work Group (EUTox): Serum indoxyl sulfate is associated with vascular disease and mortality in chronic kidney disease patients. Clin J Am Soc Nephrol 2009;4:1551-1558.

19 Taki K, Tsuruta Y, Niwa T: Indoxyl sulfate and atherosclerotic risk factors in hemodialysis patients. Am J Nephrol 2007;27:30-35. 
20 Yu M, Kim YJ, Kang DH: Indoxyl sulfate-induced endothelial dysfunction in patients with chronic kidney disease via an induction of oxidative stress. Clin J Am Soc Nephrol 2011;6:30-39.

-21 Chiu CA, Lu LF, Yu TH, Hung WC, Chung FM, Tsai IT, Yang CY, Hsu CC, Lu YC, Wang $\mathrm{CP}$, Lee YJ: Increased levels of total $p$-cresylsulphate and indoxyl sulphate are associated with coronary artery disease in patients with diabetic nephropathy. Rev Diabet Stud 2010; 7:275-284.

22 Liabeuf S, Barreto DV, Barreto FC, Meert N, Glorieux G, Schepers E, Temmar M, Choukroun G, Vanholder R, Massy ZA: Free p-cresylsulphate is a predictor of mortality in patients at different stages of chronic kidney disease. Nephrol Dial Transplant 2010;25: 1183-1191.

-23 Wang CP, Lu LF, Yu TH, Hung WC, Chiu CA, Chung FM, Yeh LR, Chen HJ, Lee YJ, Houng JY: Serum levels of total $p$-cresylsulphate are associated with angiographic coronary atherosclerosis severity in stable angina patients with early stage of renal failure. Atherosclerosis 2010;211:579-583.

-24 Meijers BK, Bammens B, De Moor B, Verbeke K, Vanrenterghem Y, Evenepoel P: Free $p$ cresol is associated with cardiovascular disease in hemodialysis patients. Kidney Int 2008;73:1174-1180.

25 Meijers BK, Claes K, Bammens B, et al: $p$-Cresol and cardiovascular risk in mild-to-moderate kidney disease. Clin J Am Soc Nephrol 2010;5:1182-1189.

-26 Scholze A, Jankowski V, Henning L, Haass W, Wittstock A, Suvd-Erdene S, Zidek W, Tepel $\mathrm{M}$, Jankowski J: Phenylacetic acid and arterial vascular properties in patients with chronic kidney disease stage 5 on hemodialysis therapy. Nephron Clin Pract 2007;107:c1-c6.

27 Spiegel DM, Raggi P, Smits G, Block GA: Factors associated with mortality in patients new to haemodialysis. Nephrol Dial Transplant 2007;22:3568-3572.

28 Chen CY, Yoshida A, Asakura M, et al: Serum blood urea nitrogen and plasma brain natriuretic peptide and low diastolic blood pressure predict cardiovascular morbidity and mortality following discharge in acute decompensated heart failure patients. Circ J 2012;76:2372-2379.

-29 Alderman MH, Cohen H, Madhavan S, Kivlighn S: Serum uric acid and cardiovascular events in successfully treated hypertensive patients. Hypertension 1999;34:144-150.

- 30 Fang J, Alderman MH: Serum uric acid and cardiovascular mortality the NHANES I epidemiologic follow-up study, 1971-1992. National Health and Nutrition Examination Survey. JAMA 2000;283:2404-2410.

- 31 Verdecchia P, Schillaci G, Reboldi G, Santeusanio F, Porcellati C, Brunetti P: Relation between serum uric acid and risk of cardiovascular disease in essential hypertension. The PIUMA study. Hypertension 2000;36:10721078.
32 Goicoechea M, de Vinuesa SG, Verdalles U, et al: Effect of allopurinol in chronic kidney disease progression and cardiovascular risk. Clin J Am Soc Nephrol 2010;5:1388-1393.

-33 Kanbay M, Huddam B, Azak A, et al: A randomized study of allopurinol on endothelial function and estimated glomerular filtration rate in asymptomatic hyperuricemic subjects with normal renal function. Clin J Am Soc Nephrol 2011;6:1887-1894.

-34 Puschett JB, Agunanne E, Uddin MN: Emerging role of the bufadienolides in cardiovascular and kidney diseases. Am J Kidney Dis 2010;56:359-370.

- 35 Kennedy DJ, Vetteth S, Periyasamy SM, et al: Central role for the cardiotonic steroid marinobufagenin in the pathogenesis of experimental uremic cardiomyopathy. Hypertension 2006;47:488-495.

36 Blacher J, Guerin AP, Pannier B, Marchais SJ, London GM: Arterial calcifications, arterial stiffness, and cardiovascular risk in end-stage renal disease. Hypertension 2001;38:938-942.

37 Bonomini M, Dottori S, Amoroso L, Arduini A, Sirolli V: Increased platelet phosphatidylserine exposure and caspase activation in chronic uremia. J Thromb Haemost 2004;2: 1275-1281.

38 Ito S, Osaka M, Higuchi Y, Nishijima F, Ishii $\mathrm{H}$, Yoshida M: Indoxyl sulfate induces leukocyte-endothelial interactions through up-regulation of E-selectin. J Biol Chem 2010;285: 38869-38875.

39 Schepers E, Glorieux G, Dou L, Cerini C, Gay$\operatorname{rard} \mathrm{N}$, Louvet L, Maugard C, Preus P, Rodriguez-Ortiz M, Argiles A, Brunet P, Cohen G, Jankowski J, Jankowski V, Massy Z, Rodriguez M, Vanholder R: Guanidino compounds as cause of cardiovascular damage in chronic kidney disease: an in vitro evaluation. Blood Purif 2010;30:277-287.

40 Bugnicourt JM, Da SC, Bengrine A, Godefroy O, Baumbach G, Sevestre H, Bode-Boeger SM, Kielstein JT, Massy ZA, Chillon JM: Chronic renal failure alters endothelial function in cerebral circulation in mice. Am J Physiol Heart Circ Physiol 2011;301:H1143H1152.

-41 Tumur Z, Niwa T: Indoxyl sulfate inhibits nitric oxide production and cell viability by inducing oxidative stress in vascular endothelial cells. Am J Nephrol 2009;29:551-557.

- 42 Tumur Z, Shimizu H, Enomoto A, Miyazaki $\mathrm{H}$, Niwa $\mathrm{T}$ : Indoxyl sulfate upregulates expression of ICAM-1 and MCP-1 by oxidative stress-induced NF-kappaB activation. Am J Nephrol 2010;31:435-441.

43 Masai N, Tatebe J, Yoshino G, Morita T: Indoxyl sulfate stimulates monocyte chemoattractant protein-1 expression in human umbilical vein endothelial cells by inducing oxidative stress through activation of the $\mathrm{NADPH}$ oxidase-nuclear factor-kappaB pathway. Circ J 2010;74:2216-2224.
44 Kharait S, Haddad DJ, Springer ML: Nitric oxide counters the inhibitory effects of uremic toxin indoxyl sulfate on endothelial cells by governing ERK MAP kinase and myosin light chain activation. Biochem Biophys Res Commun 2011;409:758-763.

45 Miyamoto $\mathrm{Y}$, Watanabe $\mathrm{H}$, Otagiri $\mathrm{M}$, Maruyama T: New insight into the redox properties of uremic solute indoxyl sulfate as a pro- and anti-oxidant. Ther Apher Dial 2011;15:129-131.

46 Shimoishi K, Anraku M, Kitamura K, Tasaki Y, Taguchi K, Hashimoto M, Fukunaga E, Maruyama T, Otagiri M: An oral adsorbent, AST-120 protects against the progression of oxidative stress by reducing the accumulation of indoxyl sulfate in the systemic circulation in renal failure. Pharm Res 2007;24:12831289.

47 Peng YS, Lin YT, Chen Y, Hung KY, Wang SM: Effects of indoxyl sulfate on adherens junctions of endothelial cells and the underlying signaling mechanism. J Cell Biochem 2012;113:1034-1043.

48 Adelibieke Y, Shimizu H, Muteliefu G, Bolati $D$, Niwa T: Indoxyl sulfate induces endothelial cell senescence by increasing reactive oxygen species production and p53 activity. J Ren Nutr 2012;22:86-89.

49 Adijiang A, Higuchi Y, Nishijima F, Shimizu $\mathrm{H}$, Niwa T: Indoxyl sulfate, a uremic toxin, promotes cell senescence in aorta of hypertensive rats. Biochem Biophys Res Commun 2010;399:637-641.

50 Faure V, Dou L, Sabatier F, Cerini C, Sampol J, Berland Y, Brunet P, Dignat-George F: Elevation of circulating endothelial microparticles in patients with chronic renal failure. J Thromb Haemost 2006;4:566-573.

51 Recht PA, Tepedino GJ, Siecke NW, Buckley MT, Mandeville JT, Maxfield FR, Levin RI: Oxalic acid alters intracellular calcium in endothelial cells. Atherosclerosis 2004;173:321328.

52 Levin RI, Kantoff PW, Jaffe EA: Uremic levels of oxalic acid suppress replication and migration of human endothelial cells. Arteriosclerosis 1990;10:198-207.

53 Moe SM, Chen NX: Pathophysiology of vascular calcification in chronic kidney disease. Circ Res 2004;95:560-567.

54 Yamamoto H, Tsuruoka S, Ioka T, Ando H, Ito C, Akimoto T, Fujimura A, Asano Y, Kusano E: Indoxyl sulfate stimulates proliferation of rat vascular smooth muscle cells. Kidney Int 2006;69:1780-1785.

55 Muteliefu G, Enomoto A, Niwa T: Indoxyl sulfate promotes proliferation of human aortic smooth muscle cells by inducing oxidative stress. J Ren Nutr 2009;19:29-32.

56 Adijiang A, Goto S, Uramoto S, Nishijima F, Niwa T: Indoxyl sulphate promotes aortic calcification with expression of osteoblast-specific proteins in hypertensive rats. Nephrol Dial Transplant 2008;23:1892-1901. 
57 Maizel J, Six I, Slama M, Tribouilloy C, Sevestre $\mathrm{H}$, Poirot S, Giummelly P, Atkinson J, Choukroun G, Andrejak M, Kamel S, Maziere JC, Massy ZA: Mechanisms of aortic and cardiac dysfunction in uremic mice with aortic calcification. Circulation 2009;119:306-313.

- 58 Lindsay RM, Prentice CR, Davidson JF, Burton JA, McNicol GP: Haemostatic changes during dialysis associated with thrombus formation on dialysis membranes. BMJ 1972;4: 454-458.

61 Radulescu D, Ferechide D: The importance of residual renal function in chronic dialysed patients. J Med Life 2009;2:199-206.

62 Lesaffer G, De SR, Lameire N, Dhondt A Duym P, Vanholder R: Intradialytic removal of protein-bound uraemic toxins: role of solute characteristics and of dialyser membrane. Nephrol Dial Transplant 2000;15:50-57.

63 Dhondt A, Vanholder R, Van BW, Lameire N The removal of uremic toxins. Kidney Int

59 Meijers BK, Van Kerckhoven S, Verbeke K, Dehaen W, Vanrenterghem Y, Hoylaerts MF, Evenepoel P: The uremic retention solute $p$ cresyl sulfate and markers of endothelial damage. Am J Kidney Dis 2009;54:891-901.

60 Bammens B, Evenepoel P, Keuleers H, Verbeke K, Vanrenterghem Y: Free serum concentrations of the protein-bound retention solute $p$-cresol predict mortality in hemodialysis patients. Kidney Int 2006;69:10811087. 2001;78:S292-S297.

65 Maduell F, Moreso F, Pons M, et al: High-efficiency postdilution online hemodiafiltration reduces all-cause mortality in hemodialysis patients. J Am Soc Nephrol 2013;24:487497.

66 Stinghen AE, Goncalves SM, Bucharles S, et al: Sevelamer decreases systemic inflammation in parallel to a reduction in endotoxemia. 2000;76:S47-S59.

67 Yamamoto S, Zuo Y, Ma J, Yancey PG, Hunley TE, Motojima M, Fogo AB, Linton MF, Fazio S, Ichikawa I, Kon V: Oral activated charcoal adsorbent (AST-120) ameliorates extent and instability of atherosclerosis accelerated by kidney disease in apolipoprotein Edeficient mice. Nephrol Dial Transplant 2011; 26:2491-2497.

68 Nakamura T, Kawagoe Y, Matsuda, Ueda Y, Shimada N, Ebihara I, Koide H: Oral adsorbent AST-120 decreases carotid intima-media thickness and arterial stiffness in patients with chronic renal failure. Kidney Blood Press Res 2004;27:121-126.

69 Shibahara H, Shibahara N: Cardiorenal protective effect of the oral uremic toxin absorbent AST-120 in chronic heart disease patients with moderate CKD. J Nephrol 2010; 23:535-540. Blood Purif 2010;29:352-356.
Humes HD, Fissell WH, Tiranathanagul K: The future of hemodialysis membranes. Kidney Int 2006;69:1115-1159. 